\title{
Mental Health Engagement Network: Innovating Community-Based Mental Healthcare
}

\author{
Forchuk C*, Rudnick A, Hoch J, Donelle L, Campbell R, Osaka W, Edwards B, Osuch E, Norman R, Vingilis E, Mitchell B, Reiss J, Corring \\ D, Petrenko M, Godin M, Reed J and McKillop M
}

Lawson Health Research Institute, Canada

\begin{abstract}
Background: Canadian mental health care reform calls for new service delivery models that capitalize on health promotion, support and early intervention as patients and services are transitioning from institutions to communities. The Mental Health Engagement Network (MHEN) intervention is a smart technology enabled service delivery model that electronically links individuals to their health care professionals, promoting information sharing between individuals and their health care professionals, and promoting access to mental health care services. This project, funded by Canada Health Infoway, began in September 2011 and will complete in March 2013.

Methods: The MHEN project is a longitudinal, mixed qualitative and quantitative research study which has recruited 400 ( 245 men and 155 women) research participants diagnosed with a mood or a psychotic disorder who are currently working with community based mental health care professionals ( 54 mental health care professionals across 4 agencies in the London and surrounding area). Each participant has been randomly assigned into Group 1 (early intervention) or Group 2 (later intervention). Group 1 participants received an iPhone 4S, a TELUS health space $^{\mathrm{TM}}$ account, and version 1.0 of the Lawson SMART record (a web-based application that provides individuals with a personal health record and tools to help them manage their health) in July, 2012. Participants in Group 2 initially acted as a control group, and received the version 2 intervention in March, 2013.

Results: Participants felt the Lawson SMART record was quite $(33.1 \%)$ or extremely $(29.2 \%)$ helpful, and gave participants quite a bit more (26.8\%) and an extreme amount more (21\%) independence. Web analytics demonstrated that participants visited the Lawson SMART record mobile and desktop home page a total of 16, 928 times.

Conclusion: This new service delivery model has the potential to provide quality care to those living in the community with mental illness, enhance health status and quality of life, and reduce the burden of mental illness on the healthcare system by decreasing more costly service uses.
\end{abstract}

\section{Introduction}

\section{The state of mental healthcare}

The mental health care system in Canada is over-burdened, underfunded and highly unorganized despite attempts to move mental illness out of the shadows and onto the radar of policy and decision-makers [1-3].

In spite of efforts to optimize service models, mental illness remains as one of the biggest contributors to healthcare spending in Canada. In fact, the Public Health Agency of Canada reported that neuropsychiatric disorders had the highest expenditure for direct health services and the third-highest expenditure for indirect costs to the economy, related to illness, disability or premature death [4]. In 2008, the Canadian economic burden of mental illness in Canada was estimated to be $\$ 51$ billion annually [5], a nearly 7 -fold increase from a 1993 analysis that estimated the burden to be around $\$ 7.3$ billion [6]. Forecasts approximate that direct costs related to mental illness over the next 30 years are likely to exceed $\$ 2.3$ trillion [7]. Despite these high costs, only one third of those who seek mental health services in Canada actually receive them [8]. As the accountability of the public sector increases, fiscal pressures will surely drive change in future healthcare spending. It is of utmost importance that these changes encompass evidence-informed solutions that will reduce the economic burden of mental illness without compromising the health and wellbeing of mental health consumers.

\section{Health services delivery}

Mental healthcare services remain fragmented and under-equipped to effectively accommodate those currently in the system. With patients and mental health care services transitioning from institutions to communities, new service delivery models are required to ensure that the Canadian mental health care system will be sustainable. Policy makers must look to new methods of delivering care that capitalize on health promotion, support and early intervention. An innovative and sustainable mode of care delivery is the use of smart technologies to enhance treatment, build capacity, and empower clients to take control of their own mental health. A new approach of delivering patientcentered care using technology has been proved to be effective in physical health promotion [9-13], and in the last decade this concept has shown great potential in psychiatric care. Web-based interventions such as online learning programs, Personal Health Records (PHR) and online databases with access to mental health resources have all generated positive outcomes for individuals with mental illness.

*Corresponding author: Dr. Cheryl Forchuk, Lawson Clinical Research Leader Mental Health Engagement Network, Canada, Tel: 519.685-8500 x.77034; E-mail: cforchk@uwo.ca

Received October 11, 2013; Accepted December 30, 2013; Published January 07, 2014

Citation: Forchuk C, Rudnick A, Hoch J, Donelle L, Campbell R, et al. (2014) Mental Health Engagement Network: Innovating Community-Based Mental Healthcare. J Gen Pract 2: 143. doi: 10.4172/2329-9126.1000143

Copyright: (c) 2014 Forchuk C, et al. This is an open-access article distributed under the terms of the Creative Commons Attribution License, which permits unrestricted use, distribution, and reproduction in any medium, provided the original author and source are credited. 
One such study employing a web-based intervention, Anxiety Online, found that providing information about anxiety disorders, links to useful resources, psychological assessments and a referral system increased access to mental health care services in a cost effective and efficient way. The authors inferred that individuals who might otherwise not access traditional treatment, due to stigma, may be encouraged to access it through this technology [14]. Another study which evaluated the use of an online cognitive behavioural therapy (CBT) treatment for depression found a reduction in symptoms of depression and an improvement in clinical outcomes of those who received the intervention [15].

Mobile-based psychiatric interventions, including text-messaging and mobile applications, have also produced positive outcomes for individuals with mental health issues. Studies using a cell phone and text message-based interventions have found increases in medication adherence, as well as improvements among negative, cognitive, and global symptoms $[16,17]$, demonstrating the potential for innovative methods of delivering care in the mental health population.

\section{Proposed solution}

The Mental Health Engagement Network (MHEN) intervention, promotes health and early intervention through a smart technologyenabled model of care. Through the MHEN project, funded by Canada Health Infoway, an intervention was developed to facilitate the delivery of health care to individuals living in the community by electronically linking individuals to their mental health care professionals, promoting information sharing between individuals and their mental health care professional, and promoting access to mental health care services. The MHEN gives individuals access to their own personal health information and health maintenance tools through a mental health application, the Lawson SMART record, and the use of mobile devices. It is proposed that this new service delivery model has the potential to provide quality care to those living in the community with mental illness, enhance health status and quality of life, and reduce the burden of mental illness on the healthcare system by decreasing costly service uses.

\section{Solution overview}

The Lawson SMART record is a web-based personal health record developed by Lawson Health Research Institute team in partnership with TELUS Health, and with further collaboration with participating community mental health agencies, clinical support workers, mental health consumers, and technical advisors. The solution provides mental health clients with an up-to-date record of their health information, which they can view and manage using any smart mobile device or desktop computer connected to the internet. Each client is given their own unique username and password to access their Lawson SMART record profile, where they can securely access an active list of medications, family medical history, immunization records, allergies, mental health care professional's contact information, care plans, and crisis plans. This health information is uploaded directly from electronic health records and updates daily when any changes are made. While it can be cumbersome and time-consuming to request access to our health information, the Lawson SMART record makes this process simple for clients.

The Lawson SMART record also permits mental health clients to take an active role in the maintenance of their own health through tools and functionalities embedded within the application. The data produced from application utilization are not intended for research purposes, but rather to inform both clients and care professionals in health-management. Tools and functionalities include:

A Mood Monitor where clients can track their mood at any given time, store it electronically, and share it in real time with their participating mental health care professional

Health Journal Notes where clients can log subjective thoughts and reminders

\section{Prompts and Reminders to assist in daily living}

Ability to track physiological measures such as blood pressure, blood glucose, cholesterol, and weight secure mental health care professional messaging [18].

Participating mental health care professionals have electronic access to their client's Lawson SMART record. This access is intended to facilitate communication between mental health care professionals and clients through increased flexibility and access, not simply to monitor client's behaviors [18].

Furthermore, participants in the MHEN project receive smart phones (iPhone $4 S$ ) with a great degree of creative potential, which is quite novel in current literature. As such, clients have found inventive solutions to their own health challenges through the use of third party applications that were not anticipated by the research team. The use of smart phones and the Lawson SMART record offers an effective, comprehensive intervention to all of the participating clients.

\section{Methods}

The following section will define the methods and parameters of the MHEN project [18].

\section{Sample}

Mental health care professionals: Recruitment of mental health care professionals began in January 2012. Participating agencies include; London Health Sciences Centre, St. Joseph's Health Care (London and St. Thomas), the Canadian Mental Health Association, and WOTCH community mental health services. These organizations operate far-reaching programs that provide community care and support to those living with mental illness in London, Ontario Canada and the surrounding area. In order to participate, each mental health care professional had to have at least 8 eligible clients for participation on their caseload, and be willing to employ the intervention in their provision of care [18].

Clients: Client participants were recruited from the caseloads of the enrolled mental health care professionals. Clients met the inclusion criteria if they had been given a mood or psychosis primary diagnosis, if they were over 18 years old and under 80 years old, and if they could read and understand English. Table I displays the descriptive statistics for the client sample of the study [18] (Table 1).

\section{Study design}

The MHEN project employed a mixed methods, delayed implementation design. Participants included 54 community mental health care professionals and 400 individuals living in the community with a mood or psychosis disorder.

Individuals from the caseloads of each of the participating mental health care professionals were randomized into either Group 1 (early 
Citation: Forchuk C, Rudnick A, Hoch J, Donelle L, Campbell R, et al. (2014) Mental Health Engagement Network: Innovating Community-Based Mental Healthcare. J Gen Pract 2: 143. doi: 10.4172/2329-9126.1000143

intervention) or Group 2 (late intervention). Group 1 received the MHEN intervention first. Participants in Group 2 initially acted as a control group and received the MHEN intervention after 6 months. A comparison of the two groups at baseline was conducted and no significant differences were found (Table 1).

\section{Data collection}

Each client participant completed four individual interviews (baseline, 6, 12 and 18 months). During the individual interviews, 8 questionnaires were administered. The questionnaires included; a demographic form, the Quality of Life -Brief Version (QoL-BV), Health, Social, and Justice Service Use form, Medical Outcomes Study 36-item Form Health Survey (SF-36), the EQ-5D, the Community Integration Questionnaire, the Adult Consumer Empowerment Scale, and the Perception of Smart Technology Form [18]. In addition to participant interviews, quantitative data was collected through web analytic reporting and measured the usage of the Lawson SMART record.

Quantitative data was also collected through a mental health care professional survey. This brief survey measured usability and adoption of the MHEN intervention, the impact of the MHEN intervention to workflow, and Lawson SMART record preferences.

Qualitative data was gathered through both client and mental health care professional focus groups that took place throughout the study. The focus groups described the phenomenology of the participants by allowing them to discuss the usability and ease of adoption of the intervention, the benefits and difficulties related to technology and by allowing them to give recommendations for the future.
This initial analysis focused on data collected from the Perception of Smart Technology form, the web analytics reporting, as well as themes from the qualitative data of both the clients and the mental health care professionals. Further analyses will be reported once data collection is complete.

\section{Training}

A half-day training sessions was a prerequisite for all participants before receiving their smart phone and access to the Lawson SMART record. The training content was developed with input from consumers, mental health care professionals, and community partners with expertise in training, industry partners, and other key stakeholders to ensure that a comprehensive tutorial was developed. Each session comprised of a didactic lesson, a live demonstration with the active technology and a participatory seminar where everyone had the chance to practice, learn and ask questions while in the presence of technical support. Training participants also received an extensive instructional package that included lessons, screenshots, and walk-throughs.

With any new technology, there were anticipated and unanticipated challenges to overcome. To address these matters, drop-in training periods were scheduled weekly for participants who wanted to ask questions, expand their knowledge, and learn new things about the device and the program. Furthermore, at-home training was also made available in special circumstances where client participants were not able to attend the sessions. In addition, a Blog was developed to keep clients and mental health care professionals informed about upcoming drop-in sessions, to provide announcements about any project changes, and to link participants to helpful resources related to the intervention.

\begin{tabular}{|c|c|c|c|}
\hline Characteristic & Intervention Group n (\%) & Control Group n(\%) & Total Sample n(\%) \\
\hline Age (years) [Mean (SD)] & $38.2(14.62)$ & $37.1(12.9)$ & $37.6(13.76)$ \\
\hline \multicolumn{4}{|l|}{ Sex } \\
\hline Male & $125(65.1 \%)$ & $114(56.4 \%)$ & $239(60.7 \%)$ \\
\hline Female & $67(34.9 \%)$ & $88(43.6 \%)$ & $155(39.3 \%)$ \\
\hline \multicolumn{4}{|l|}{ Marital Status } \\
\hline Single, never married & $140(72.9 \%)$ & $136(67.3 \%)$ & $276(70.1 \%)$ \\
\hline Married/Common-law & $18(9.4 \%)$ & $15(7.4 \%)$ & $33(8.4 \%)$ \\
\hline Separated/Divorced & $32(16.7 \%)$ & $50(24.8 \%)$ & $82(20.8 \%)$ \\
\hline Widowed & $2(1.0 \%)$ & $1(0.5 \%)$ & $3(0.8 \%)$ \\
\hline Has Children & $56(29.2 \%)$ & $67(33.2 \%)$ & $123(31.2 \%)$ \\
\hline Has Contact with Family & $174(92.1 \%)$ & $190(94.1 \%)$ & $364(93.1 \%)$ \\
\hline \multicolumn{4}{|l|}{ Highest Level of Education } \\
\hline Grade school & $52(27.1 \%)$ & $66(32.8 \%)$ & $118(30.0 \%)$ \\
\hline High school & $93(48.4 \%)$ & $84(41.8 \%)$ & $177(45.0 \%)$ \\
\hline Community college/University & $46(24.0 \%)$ & $51(25.4 \%)$ & $97(24.7 \%)$ \\
\hline Currently Employed & $47(24.5 \%)$ & $50(24.8 \%)$ & $97(24.6 \%)$ \\
\hline \multicolumn{4}{|l|}{ Psychiatric Diagnoses } \\
\hline Psychotic disorder & $111(57.8 \%)$ & $123(60.9 \%)$ & $234(59.4 \%)$ \\
\hline Mood disorder & $119(62.0 \%)$ & $107(53.0 \%)$ & $226(57.4 \%)$ \\
\hline Anxiety disorder & $60(31.2 \%)$ & $64(31.7 \%)$ & $124(31.5 \%)$ \\
\hline Substance related disorder & $30(15.6 \%)$ & $20(9.9 \%)$ & $50(12.7 \%)$ \\
\hline Personality disorder & $12(6.2 \%)$ & $12(5.9 \%)$ & $24(6.1 \%)$ \\
\hline Disorder of childhood/adolescence & $9(4.7 \%)$ & $13(6.4 \%)$ & $22(5.6 \%)$ \\
\hline Other & $5(2.6 \%)$ & $6(3.0 \%)$ & $11(2.8 \%)$ \\
\hline Developmental handicap & $0(0.0 \%)$ & $3(1.5 \%)$ & $3(0.8 \%)$ \\
\hline Organic disorder & $2(1.0 \%)$ & $1(0.5 \%)$ & $3(0.8 \%)$ \\
\hline Diagnosis type unknown & $1(0.5 \%)$ & $1(0.5 \%)$ & $2(0.5 \%)$ \\
\hline
\end{tabular}

Table 1: Description of Client Participants $(n=400)$ [18] 


\section{Results}

\section{Quantitative analysis}

Results from the Perception of Smart Technology Form at the 12 month post-intervention periods indicated initial positive feedback regarding the Lawson SMART record. Data collected using the 7-point Likert scale indicated that participants felt the Lawson SMART record was 6 -quite $(33.1 \%)$ or 7 -extremely $(29.2 \%)$ helpful. It also indicated that it gave participants 6-quite a bit more $(26.8 \%)$ and 7 -an extreme amount more $(21 \%)$ independence.

The Perception of Smart Technology Form also used the 7-point delighted-terrible scale to measure how clients felt about the Lawson SMART record. Results indicated that participants were 7 -delighted $(24.1 \%)$ or 6-pleased (35.8\%) with having access to their own personal health record, whereas only a small number of participants felt 1 -terrible $(0.3 \%)$ or 2 -unhappy $(0.6 \%)$ with having access. A majority of participants indicated that they were either 7-delighted $(22.7 \%)$ or 6-pleased (31.0\%) about connecting with their mental health care professional using the Lawson SMART record, whereas only a small amount of participants felt 7-terrible (4.1\%) of 6-unhappy (3.3\%) with this function. Similarly, (26.6\%) were 7-delighted or 6-pleased $(34.2 \%)$ about being able to share their personal health information with their mental health care professionals electronically, whereas only a small number of participants felt 1-terrible (2.0\%) or 2-unhappy (3.0\%).

Web analytics from August 2012 until July 2013 demonstrated that participants visited the Lawson SMART record mobile and desktop home page a total of 16, 928 times (13202 times and 3726 times respectively). The most commonly used Lawson SMART record widgets included; the Health Journal (20,883 hits to page), Care Professional Messaging $(3,816)$, Health Journal Notes $(3,174)$, Appointments (1,660), Reminders (801) and My Daily Tasks (576).

\section{Qualitative analysis}

Participatory action is entrenched at the core of the MHEN research and involving mental health consumers, mental health care professionals, and other stakeholders in the research conversations has led to the collaborative and reflective development of this solution. The research has engaged clients and mental health care professionals in participatory action through qualitative focus groups at multiple key points throughout the study. To date, there have been 16 focus groups for clients and 7 for mental health care professionals. Participant dialogue was recorded and transcribed without identifying information. Themes among the different groups were then identified and matched to their lived experience with the intervention.

\section{Client focus groups}

When asked how care using the Lawson SMART record was different when compared to their usual care, a common theme was that it improved access to their health care professionals and impacted their information access and sharing. Clients were also asked to describe any health improvements that they've noticed since adopting the new technology. A common theme was that it increased their self-awareness and made them feel more connected to society. Since adopting this technology, many clients expressed feelings of greater control in their health management and in their life, and that they were able to function more independently.

When asked about barriers to using the Lawson SMART record, clients identified the sign in process as being onerous due to the stringent security measures. Other barriers included the application speed, lack of engagement from some care professionals, and confusion about the application being web-based, and not a typical smart phone application. Some clients also expressed frustrations with the screen size and difficulties with the sensitivity of the touch screen.

\section{Mental health care professional focus groups}

When asked how it reflected their relationship with their clients and other staff, mental health care professionals identified that clients were more accessible and that communication was more consistent. They also cited a boost in client self-esteem, clients feeling more connected, and that it created more awareness and self-reflection. Care professionals were also pleased with their clients increased ability to track and manage their appointments and organize personal health goals. Time savings in case management was another commonly cited experience.

Mental health care professionals identified some barriers to incorporating the Lawson SMART record into their usual workflow. A common theme was that it was onerous to check for messages from clients, and that the sign in process was quite slow. Furthermore, care professionals expressed some frustrations with troubleshooting clients' technical issues that they felt wasn't part of their role.

\section{Discussion}

Preliminary results indicated that the MHEN service model has the potential to improve healthcare delivery in the future. The data suggests that both clients and mental health care professionals were adopting the Lawson SMART record into their care. Clients have reported that the intervention was helpful and granted them more independence in their care. Themes from the qualitative analysis indicated that it improved access to their care professionals, and increased self-awareness and their connection to society. Care professionals reported an improvement in their clients' health and indicated a positive impact on certain areas of workflow. Technical analysis also demonstrated that clients frequently visited their Lawson SMART record throughout the study. Similar themes in limitations of the intervention were apparent from both clients and care professionals. Common themes that hindered application use were difficulties with the sign-in process, application speed, and limitations related to the screen size and sensitivity of the touch screen.

Although this initial analysis has indicated improvements in client outcomes, further data collection and analyses are needed to explore the efficacy of the intervention. A future in-depth analysis of quality of care measures, health status, empowerment, quality of life, and economic impact between the intervention and control groups are needed to investigate the impact of the intervention. Furthermore, comparisons among subgroups within the study will provide a more comprehensive understanding of the intervention's potential and how this knowledge development can best shape future models of care.

\section{Conclusion}

Technology-enabled models of care have shown to improve the quality of and access to services for clients living with mental illness. The MHEN project has built upon these concepts and created an innovative and holistic way for client and mental health care professionals to collaborate in care through the use of personal health records and smart technology. Initial results were promising and suggest the potential for an overhaul in service delivery of mental health care. Further analyses 
Citation: Forchuk C, Rudnick A, Hoch J, Donelle L, Campbell R, et al. (2014) Mental Health Engagement Network: Innovating Community-Based Mental Healthcare. J Gen Pract 2: 143. doi: 10.4172/2329-9126.1000143

Page 5 of 5

in this study are expected to be promising and will influence the way that care is delivered.

\section{References}

1. McAllister J (2009) The healthcare interview: Micheal Kirby breaks down barriers to better mental health care". Canadian Health Care Network.

2. Statistics Canada (2003) Canadian community health survey: Mental health and well-being

3. Mental Health Commission of Canada (2013) Making the case for investing in mental health in Canada.

4. Public Health Agency of Canada (2009) Investing in prevention - The economic perspective: Key findings from a survey of the recent evidence.

5. Lim K-L, Jacobs P, Ohimnaa A, Schopflocher D, Dewa CS (2008) A new population-based measure of the economic burden of mental illness in Canada. Chronic Dis Can 28: 92-98.

6. Health Canada (2002) A report on mental illness in Canada. Ottawa, Canada.

7. Smetanin $P$, Stiff D, Briante C, Adair CE, Ahmad S et al. (2011) The life and economic impact of major mental illnesses in Canada: 2011 to 2041. Risk Analytica.

8. Statistics Canada (2003) Canadian community health survey: Mental health and well-being.

9. Mattila E, Korhonen I, Salminen JH, Ahtinen A, Koskinen E, et al. (2010) Empowering citizens for well-being and chronic disease management with wellness diary. IEEE Trans Inf Technol Biomed 14: 456-463.
10. Lynch CP, Egede LE (2011) Optimizing diabetes self-care in low literacy and minority populations: Problem-solving, empowerment, peer support and technology-based approaches. J Gen Intern Med 26: 953-955.

11. Gubrium, A. (2009). Digital storytelling: an emergent method for health promotion research and practice. Health Promotion Practice 10: 186-191.

12. Cheitlin Cherry J, Moffatt TP, Rodriguez C, Dryden K (2002) Diabetes disease management program for an indigent population empowered by telemedicine technology. Diabetes Technol Ther 4: 783-791.

13. Moore L, Wasson J (2006) An introduction to technology for patient-centered collaborative care. J Ambul Care Manage 29: 195-198.

14. Klein B, Meyer D, Austin DW, Kyrios M (2011) Anxiety online - a virtual clinic: Preliminary outcome following completion of five fully automated treatment programs for anxiety disorders and symptoms. J Med Internet Res 13.

15. Titov N, Andrews G, Davies M, McIntyre K, Robinson E, et al. (2010) Internet treatment for depression: A randomized controlled trial comparing clinician vs. technician assistance. PLoS ONE 5.

16. Granholm E, Ben-Zeev D, Link P, Bradshaw K, Holden J (2011) Mobile Assessment and Treatment for Schizophrenia (MATS): a pilot trial of an interactive text-messaging intervention for medication adherence, socialization and auditory hallucinations. Schizophr Bull 38

17. Montes JM, Medina E, Gomez-Beneyto M, Maurino J (2012) A short message service (SMS)-based strategy for enhancing adherence to antipsychotic medication in schizophrenia. Psychiatry Res 200: 89-95.

18. Forchuk C, Rudnick A, Hoch J, Donelle L, Reiss J, et al. (2013) Mental health engagement network (MHEN). International Journal on Advances in Life Sciences 51-10. 